\title{
Zur lexikalischen Semantik des Deutschen ${ }^{1}$
}

\author{
Winfried Ulrich ${ }^{*}$
}

Zusammenfassung: In diesem Aufsatz wird das mentale Lexikon als System beschrieben, in dem Lexeme aufgenommen, gespeichert und nach bestimmten Regeln geordnet und eingesetzt werden. Hierbei spielen Bedeutungsbeziehungen eine grundlegende Rolle. Die Mikrostruktur dieses Netzwerks bildet die Polysemie, die durch Bedeutungssterne (mit Kernbedeutung und Nebenbedeutungen einzelner Lexeme) dargestellt werden kann. Anhand von Musterübungen wird gezeigt, wie dieses Thema im muttersprachlichen Unterricht didaktisch umgesetzt werden kann.

Stichwörter: Semantik, Lexem, Polysemie, Didaktik, Wortschatzarbeit.

Resumo: O presente trabalho descreve o léxico mental enquanto sistema no qual os lexemas são assimilados e armazenados. As relações de significado têm uma importância fundamental para as regras que influenciam a organização e o uso dos lexemas. A microestrutura dessa rede é formada pela polissemia, que pode ser representada através de "estrelas" de significados (em que o significado prototípico do lexema ocupa a posição central e os significados secundários posições periféricas). São apresentados alguns exercícios-modelo para ilustrar como este tema pode ser abordado em sala de aula.

Palavras-chaves: Semântica, lexema, polissemia, didática, exercícios de vocabulário.

\begin{abstract}
This paper describes the mental lexicon as a system in which the lexemes are assimilated, memorized, organized and applied according to certain rules. The microstructure of this network is based on polysemy which can be represented by star graphs (showing the prototypical and the secondary meanings of a lexeme). Some sample exercises are presented in order to illustrate the practical application in schools.
\end{abstract}

Key-words: Semantics, lexeme, polysemy, didactics, vocabulary exercises.

\footnotetext{
${ }^{1}$ Es handelt sich bei diesem Beitrag um die überarbeitete Fassung eines Vortrags, den der Verfasser am 18. März 2010 an der Universidade de São Paulo, Departamento de Letras Modernas, gehalten hat.

*Christian-Albrechts-Universität zu Kiel. Email: ulrich@germsem.uni-kiel.de
} 
Ulrich, W. - Zur lexikalischen Semantik des Deutschen

\section{Wort und Lexem}

Was ist ein Wort? Wörter bilden die elementare Grundlage aller menschlichen Sprache. Für viele Menschen sind Wörter schlicht die Sprache, das menschliche Verständigungsmittel schlechthin, sei es das gesprochene oder das geschriebene Wort.

Eltern freuen sich, wenn ihr Kind nach einer langen Vorphase des kindlichen Spracherwerbs etwa am Ende des ersten Lebensjahres sein erstes erkennbares Wort ausspricht und damit in aller Regel jemanden (z. B. Mama) oder etwas (z. B. Wauwau) bezeichnet. Es hat dann gelernt, dass seine Bezugspersonen mit einer bestimmten Laut- oder Phonemverbindung /ma:ma:/ oder /mama/ und /waowao/ eine bestimmte Bedeutung, 'Mutter' und 'Hund', fest verknüpft haben und dass man mit diesem akustisch wahrnehmbaren Zeichen auf etwas außerhalb des Zeichens verweisen, auf etwas zeigen kann, ohne dafür den Zeigefinger zu benutzen: eine Person, ein Tier, ein Ding, einen Sachverhalt in der außersprachlichen Erfahrungswelt (vgl. SZAGUN 2006: 114ff.) oder in einer davon abgeleiteten fiktiven Welt (z. B. Zauberer).

Zeichentheoretisch ausgedrückt: Ein von Menschen gemachtes Zeichen, mit dem man auf etwas in unserer realen oder nur vorgestellten Welt verweisen kann, beruht bekanntlich immer auf einer Vereinbarung, nach der mit einem sinnlich wahrnehmbaren Signal eine bestimmte Information verknüpft ist. Ein Sprachzeichen besteht immer materiell aus einer bestimmten Folge von Lauten bzw. Buchstaben (der Form) und immateriell der damit verknüpften Bedeutung (dem Inhalt). Die Bedeutung ist ein menschlicher Bewusstseinsinhalt, eine geistig-begriffliche Vorstellung von etwas - in der Psychologie spricht man von „Konzepten“ -, nicht etwa der mit dem Sprachzeichen bezeichnete Gegenstand oder Sachverhalt selbst, auf den der Sprecher oder Schreiber sich bezieht (Referenzobjekt). So verweisen z. B. die Wörter Abendstern und Morgenstern auf dasselbe Referenzobjekt (Planet Venus), sind aber nicht bedeutungsgleich: 'am Abend zu sehen' - 'am Morgen zu sehen'. Bedeutung ist $\mathrm{zu}$ verstehen als sprachspezifischer Bewusstseinskomplex, dem eine sprachliche Form zugeordnet ist.

Bei der Konzeptualisierung, der kognitiven Verarbeitung und Ordnung menschlicher Sinneswahrnehmungen zu Vorstellungen/Konzepten verfahren die Mitglieder verschiedener Kulturkreise und Sprachgemeinschaften aufgrund gleicher Sinnesorgane ähnlich, aber nicht immer gleich. Man kann die Welt unterschiedlich sehen und interpretieren, wie das bekannte 
Ulrich, W. - Zur lexikalischen Semantik des Deutschen

Beispiel vom halb vollen oder halb leeren Glas zeigt. Und man kann die Konzepte unterschiedlich verbalisieren. So wird im Deutschen Suppe grundsätzlich gegessen (man benutzt ja schließlich einen Löffel), im Türkischen dagegen getrunken (sie ist ja schließlich flüssige Nahrung). Beide Konzepte und beide Versprachlichungen erscheinen gleichermaßen plausibel. Dabei gibt es aber durchaus nicht für alle Konzepte auch sprachliche Zeichen. Wer im Deutschen seinen Hunger gestillt hat, also nicht mehr hungrig ist, ist satt. Was aber ist derjenige, der seinen Durst gestillt hat, nicht mehr durstig ist? Hier klafft im Deutschen eine lexikalische Lücke. Offenbar ist der kommunikative Bedarf an einem speziellen Wort, das diesen Zustand bezeichnet, nicht groß genug. Der aus einem Preisausschreiben hervorgegangene Vorschlag sitt hat sich jedenfalls nicht durchgesetzt.

Bei genauerer Betrachtung erweisen Wörter sich als hochkomplexe Gebilde. Versuche zu definieren, was genau ein Wort ist, stoßen auf eine Reihe von Schwierigkeiten. Intuitiv neigt man meist $\mathrm{zu}$ einer (ortho-)graphischen Begriffsbestimmung: Wort als bestimmte verbundene Buchstabenfolge/Graphemfolge, die auf beiden Seiten durch Leerstellen/Abstände oder durch ein Satzzeichen vom Kontext abgegrenzt ist. Sieht man einmal davon $a b$, dass die Schrift gegenüber der gesprochenen Sprache nur das Sekundärsystem ist und dass den Abständen zwischen den geschriebenen Wörtern keineswegs immer eine Sprechpause entspricht, so fallen auch in der geschriebenen Sprache bereits die trennbaren Präfixverben aus dem Rahmen: Man wird wohl kaum den Infinitiv auslachen als ein Wort, die flektierte Form lacht aus aber als zwei Wörter ausgeben können. Und Flexionsformen der Nennform lachen wie lache, lachst, lacht, lachten, gelacht sind natürlich nur unterschiedliche grammatische Formen eines Wortes und nicht mehrere Wörter, da die referenzielle Bedeutung gleich bleibt.

Auch semantische Bestimmungsversuche führen zu Problemen. Sieht man das Wort als die kleinste selbstständig Bedeutung tragende Einheit der Sprache an, so stößt man nicht nur auf die schon erwähnte Konkurrenz mit dem Morphem, sondern sucht auch anders als bei den Inhaltswörtern (Substantive, Verben, Adjektive) bei den Form- oder Funktionswörtern, die die grammatisch-logischen Beziehungen der Wörter im Satz untereinander oder im Text bestimmen, nach einer Bedeutung (der, ein, und, an, sie). Auch sind die Polysemie eines einzigen Wortes (z. B. die Bedeutungen von Läufer: 'Sportler', 'Schachfigur', 'textiler Bodenbelag') und die Homonymie verschiedener Wörter (z. B. Tau 1 als 'Niederschlag' und $\mathrm{Tau}_{2}$ als 'Seil') oft sehr schwer zu unterscheiden. 
Ulrich, W. - Zur lexikalischen Semantik des Deutschen

Es gibt also Wortbeispiele, die sich allen Definitionsversuchen widersetzen. Häufig geht man darüber allerdings großzügig hinweg und legt bei der Verwendung des überall geläufigen Begriffes Wort weniger strenge Maßstäbe an als sonst in der Linguistik üblich. Man geht dann davon aus, dass ein Wort verschiedene Merkmale aufweist, die aber nicht in jedem Falle alle vorhanden sein müssen. Unter den mehr oder weniger typischen Wörtern hat dann nur das ,prototypische“ Wort in jeder Hinsicht Wortcharakter:

- Es ist in mündlicher Rede und in der Schrift isolierbar.

- Es ist selbstständiger Bedeutungsträger.

Es besteht entweder aus einem einzigen freien Morphem und gilt dann als ,einfaches Wort“ (Simplex), oder es besteht aus mehreren Morphemen und gilt dann als „komplexes Wort“.

Ein anderer, in der Linguistik bevorzugter Terminus, der sich teilweise auf die gleichen Erscheinungen bezieht wie der Wortbegriff, allerdings unter dem Gesichtspunkt der Einordnung in den gesamten Wortschatz einer Sprache, ist das „Lexem“. Der Wortschatz, also die Menge aller zu einer bestimmten Zeit verwendeten Sprachzeichen einer Sprache oder Sprachgemeinschaft, wird als das „Lexikon“ der Sprache bezeichnet; die Grundeinheit des Lexikons ist das Lexem, womit alle benennenden und verallgemeinernden Wortschatzelemente als semantische Ganzheiten erfasst werden. Als Lexikoneinheiten sind Lexeme gesellschaftlich gefestigt und lexikalisiert. Nach Form und Bedeutung lassen sich drei Arten von Lexemen unterscheiden:

- einfache Wörter, die aus einem einzigen freien Morphem (Verben aber mit zusätzlichem Flexionsmorphem) bestehen (Haus, hoch, lauf-),

- komplexe Wörter, die durch Wortbildung aus mehreren Morphemen entstanden sind, also Zusammensetzungen (Hochhaus), Ableitungen (verlaufen, Läufer), Kurzformen (Bus, Bafög, U-Bahn als komplexes Wort mit einem Kurzwort),

- Wortgruppenlexeme, nämlich Redewendungen als feste Wortverbindungen, deren Bedeutungen nicht einfach aus den Bedeutungen der Einzelwörter abgeleitet werden kann (nicht alle Tassen im Schrank haben mit der Bedeutung 'verrückt sein'); Wortgruppenlexeme werden auch „Phraseologismen“ (oder kurz: „Phraseme“) genannt.

Die einfachen Wörter sind auf synchronischer Ebene arbiträre Zeichen, mit Ausnahme der wenigen lautmalerischen Wörter unmotiviert und deshalb als konventionelle Verknüpfung 
Ulrich, W. - Zur lexikalischen Semantik des Deutschen

von Form und Inhalt ohne besondere Merkhilfen vom Lerner einer Sprache schlicht einzuprägen.

Die komplexen Wörter dagegen sind (jedenfalls zum Zeitpunkt ihrer Bildung und ihres ersten Gebrauchs) motiviert, d. h. man kann ihre Bedeutung wenigstens teilweise auf die Bedeutungen ihrer Bestandteile zurückführen: Eine Haustür z. B. ist zwar nicht einfach die Summe von Haus und Tür (nicht jede Tür des Hauses ist schließlich eine Haustür), aber sie ist eine Tür, und sie hat etwas mit dem Haus zu tun (führt als Haupteingang in das Haus hinein). Wer die beiden einfachen Wörter schon kennt, kann sich auf deren Bedeutungen stützen, wenn er das zusammengesetzte Wort erlernt. Freilich haben viele komplexe Wörter im Laufe ihrer Geschichte an morphologischer (Adler $\leftarrow$ edel ar) und/oder semantischer Durchsichtigkeit eingebüßt (ein Handschuh ist kein Schuh) oder sie ganz verloren (Gift = Ableitung von geben mit der älteren Bedeutung 'Gabe'). Dennoch stellen durch Wortbildung entstandene Wörter auch bei nur noch gradueller Motiviertheit eine Klasse von Lexemen dar, bei deren Erwerb und Gebrauch Kenntnisse der deutschen Wortbildungsregeln eine große Hilfe sind.

Der dritte Lexemtyp besteht aus mehreren Wörtern, muss aber im Unterschied zu freien Syntagmen (deren Bedeutung vollständig aus den Bedeutungen ihrer Bestandteile und deren syntaktischer Kombination abgeleitet werden kann, z. B. nicht alle Tassen auf den Tisch stellen) wie die anderen Lexeme von den Mitgliedern der Sprachgemeinschaft als semantische Ganzheit mit seiner speziellen Bedeutung gelernt und im Gedächtnis gespeichert werden. Prototypische Wortgruppenlexeme sind stabil; ein Austausch von Bestandteilen (*nicht alle Untertassen im Schrank haben) oder eine Erweiterung (*nicht alle Teller und Tassen im Schrank haben) sind nur sehr bedingt möglich. Als gespeicherte „Fertigteile“ liegen Wortgruppenlexeme zum Gebrauch bereit und müssen nicht wie die freien Syntagmen ständig neu gebildet werden. Einige Redewendungen sind heute völlig unmotiviert (jemandem einen Bären aufbinden), viele aber wenigstens noch teilmotiviert: Wenn jemand etwas sich besonders gut merken soll und man für diese Aufforderung die Wendung sich etwas hinter die Ohren schreiben benutzt, dann deuten die Bestandteile Ohren (man muss die Ohren aufsperren und gut hinhören, was man sich merken soll) und schreiben (man schreibt sich oft auf, was man nicht vergessen will) noch schwach die Bedeutungsrichtung an.

Die partielle Motivierteit der komplexen Wörter und der Phraseme spielt beim Erwerb des mentalen Lexikons eine wichtige Rolle. Man kann sogar sagen, dass jede Sprache ein 
Ulrich, W. - Zur lexikalischen Semantik des Deutschen

ausgewogenes Verhältnis von arbiträren, unmotivierten Lexemen einerseits und teilmotivierten Lexemen andererseits aufweisen muss, um erlernbar zu sein und zu bleiben. Ein bestimmtes Maß an Motiviertheit darf nicht unterschritten werden. Sprachgeschichtlich ist zwar eine Tendenz zur allmählichen Demotivation festzustellen (Wortbildungsprodukte tendieren als Benennungseinheiten zu einer ganzheitlichen Semantik), doch steht diesem Motivationsverlust ein ständiger Motivationszuwachs durch die Bildung von neuen komplexen Wörtern (Neologismen) gegenüber, und lernpsychologisch gesehen sollte man dieser Tendenz durch erhellendes Durchleuchten der undurchsichtig werdenden Lexeme im Unterricht entschieden entgegenwirken.

Oft fast noch wichtiger als die von den Bestandteilen gestützte denotative Bedeutung der Neologismen (mit Hilfe ihrer Bedeutungsmerkmale zu bestimmen) sind dabei freilich die Konnotationen. Konnotationen sind mit einem Lexem mitverstandene Zusatzinformationen über die Verwendung von Lexemen, z. B. stilistische (gehoben, umgangssprachlich, salopp, vulgär) Markierungen.

\section{Wortschatz und mentales Lexikon}

„Wortschatz“ und „Lexikon“ sind dann synonyme und gegeneinander austauschbare Begriffe, wenn wir darunter das Inventar, die Gesamtmenge aller Lexeme einer Sprache oder Sprachgemeinschaft (zu einem bestimmten Zeitpunkt) fassen, also auch die Wortgruppenlexeme. Die Gesamtzahl lässt sich nur schwer schätzen. In der Fachliteratur schwanken die Angaben zwischen 300.000 und 500.000 Lexemen in der deutschen Standardsprache (RÖMER/MATZKE 2003: 38). Das „Deutsche Wörterbuch“ der Brüder Grimm verzeichnet etwa 500.000 Wörter. Bei Berücksichtigung der Fachsprachen liegt man allerdings angeblich bei 5 bis 10 Millionen (RÖMER/MATZKE 2003: 38). Außerdem handelt es sich historisch betrachtet um einen sich laufend verändernden Bestand: Einige Lexeme kommen mit der Zeit außer Gebrauch (Fräulein), veralten (Oheim) und verschwinden aus dem Gedächtnis der Sprachbenutzer (Magen für 'Verwandte'), andere, bedeutend mehr, kommen ständig neu hinzu (Friedwald, herunterladen, Kita, SMS, Wegfahrsperre, Waschbrettbauch, , Parteispenden-Untersuchungsausschuss, Schwangerschaftskonfliktberatung). 
Ulrich, W. - Zur lexikalischen Semantik des Deutschen

Kein Mitglied der Sprachgemeinschaft beherrscht den gesamten deutschen Wortschatz, sämtliche allgemein gebräuchlichen (also nicht nur wie z. B. Netzbürger und Neufünfland als Augenblicksbildungen/Okkasionalismen auftauchenden und sofort wieder verschwindenden) Wörter und Phraseologismen. Jedes Individuum erlernt von Kindesbeinen an bis zum Tode nur einen bescheidenen Teil und speichert ihn im Langzeitgedächtnis. Man bezeichnet diesen individuellen Anteil, genauer: dessen psychische Repräsentation, als das innere oder mentale Lexikon eines Menschen („menschlicher Wortspeicher“, AITCHISON 1997: 44; ausführliche Beschreibung bei ENGELKAMP 1995). Der Bestand an Lexemen lässt sich auch beim mentalen Lexikon nur schätzen. Immerhin kann man feststellen, dass ein Kind nach dem ersten wiedererkennbaren Wort zu Beginn des zweiten Lebensjahres im Alter von einundeinhalb Jahren durchschnittlich schon 10 bis 20 Wörter hervorbringt, mit zwei Jahren 50 oder mehr und wenige Jahre später bereits hunderte.

Man muss freilich zwischen dem immer viel umfangreicheren Verstehenswortschatz (rezeptiver Wortschatz, irreführend auch ,passiver Wortschatz“ genannt) und dem deutlich kleineren Ausdruckswortschatz (produktiver Wortschatz) unterscheiden (CLARK 1993: 245). Bei der Einschulung versteht das sechsjährige Kind bereits bis zu 14.000 Lexeme (CLARK 1995: 393) und verwendet selbst zwischen 3.000 und 4.000 oder sogar 5.000 bis 6.000 (KAUSCHKE 2000: 35). Mit dem Schriftspracherwerb erfolgt ein starker Wachstumsschub, der die ganze Schulzeit über anhält (nach CLARK 1995: 393 im englisch-amerikanischen Sprachraum bis zum 17. Lebensjahr mindestens 3.000 neu verstandene Wörter pro Jahr!), sich danach dann abschwächt. Der durchschnittliche Erwachsene speichert rezeptiv zwischen 50.000 und 100.000 Wörter, er verwendet produktiv mehrere tausend, wohl zwischen 6.000 und 10.000 (RÖMER/MATZKE 2005: 38), nach anderen Schätzungen sogar zwischen 30.000 und 40.000 Wörtern (JESCHENIAK 2002: 23) bzw. zwischen 20.000 und 50.000 Wortformen (Clark 1995: 393).

Die im mentalen Lexikon gespeicherten Informationen zu einem Lexem weisen in verschiedene Richtungen (vgl. RothWEILER/MEIBAUER 1999: 11f.). Sie beziehen sich auf phonetisch-phonologische bzw. graphematische Merkmale (Aussprache/Lautgestalt bzw. Schreibweise/Schriftgestalt), auf morphosyntaktische Merkmale (Wortart und syntaktische Verwendungsweise, Flexionsformen, Wortbildung), vor allem aber auf semantische Merkmale (Bedeutung und Bedeutungsbeziehungen sowie Referenz). Beim Lexem Schneemann wären dies z. B. phonologisch: [Jne:man], graphematisch: ১Schneemann`, 
Ulrich, W. - Zur lexikalischen Semantik des Deutschen

morphologisch: Zusammensetzung aus den beiden Basismorphemen /schnee/ und /mann/, syntaktisch: Substantiv, Plural Schneemänner, semantisch: 'aus Schnee geformte menschenähnliche Figur'. All diese Informationen werden beim sprachlichen Handeln benötigt. Wer eine Äußerung von sich geben will, wird zuerst die nichtsprachlichen Konzepte suchen, die Vorstellungen, die seiner Mitteilungsabsicht entsprechen. Es schließt sich die Versprachlichung in mehreren Schritten an: lexikalische Kodierung (Lexemwahl), grammatische Kodierung (Erstellen eines syntaktischen Bauplans unter Berücksichtung der morphosyntaktischen Merkmale der gewählten Lexeme), phonologische und prosodische bzw. graphematische Kodierung (unter Berücksichtigung der entsprechenden Lexemmerkmale), Artikulation bzw. Niederschrift. Ob diese Teilprozesse getrennt voneinander (modular/sequenziell) ablaufen oder gleichzeitig und miteinander verbunden (interaktiv), ist in der Forschung umstritten (JESCHENIAK 2002: 19-21).

Das mentale Lexikon ist keine ungeordnete Ansammlung von Lexemen, sondern ein wohlgeordnetes System, ein vieldimensionales Netzwerk, in dem alle Elemente in Beziehung zueinander stehen, vergleichbar etwa mit den durch Synapsen verbundenen Nervenzellen unseres Gehirns. Assoziationstests haben gezeigt, dass zahlreiche Angehörige derselben Sprachgemeinschaft auf ein bestimmtes Reizwort spontan mit den gleichen Reaktionswörtern antworten. Z. B. reagieren viele auf das Adjektiv eingefleischt mit dem Substantiv Junggeselle, auf Farbe mit rot, auf Handwerkzeug mit Hammer, auf heiß mit kalt. Solche überindividuell gleichen Assoziationen geben Aufschluss über die Organisation des mentalen Lexikons. Reiz- und Reaktionswort sind offenbar so fest miteinander vernetzt, dass sie sich gegenseitig herbeirufen. Mit dem einen stellt sich sogleich das andere ein.

Unter diesen engen Beziehungen zwischen Elementen des mentalen Lexikons unterscheidet man zwischen syntagmatischen und paradigmatischen Bedeutungsbeziehungen. Die syntagmatischen Relationen sind durch Verträglichkeitsbedingungen gekennzeichnet. Man registriert während des Spracherwerbs, welche Lexeme im Satz gemeinsam, koordiniert auftreten können, welche sehr oft nebeneinander erscheinen (z. B. blond, Haar, kämmen in den Syntagmen blondes Haar und das Haar kämmen), und merkt sich das; umgekehrt lernt man auch, welche Lexeme im Satz unverträglich sind (z. B. kann das Verb lachen in keinem Satz Prädikat sein, in dem das Substantiv in Subjektposition nicht das Bedeutungsmerkmal 'menschlich' aufweist: *Die Tische lachen). Solche Gebrauchsbedingungen werden als semantische Merkmale der Lexeme gespeichert. 
Ulrich, W. - Zur lexikalischen Semantik des Deutschen

Die paradigmatischen Bedeutungsrelationen verweisen nicht auf das Nebeneinander, auf das Sowohl-als-Auch von Lexemen im Satz, sondern auf ein Entweder-Oder. Immer nur eins der paradigmatisch vernetzten Lexeme kann im Satz stehen; es kann nur gegen ein anderes ausgetauscht werden. Der Sprecher oder Schreiber kann und muss also auswählen, welcher Ausdruck aus einer ganzen Reihe von möglichen Lexemen seiner Ausdrucksabsicht am besten gerecht wird.

$\mathrm{Zu}$ den paradigmatischen Beziehungen gehören die Äquivalenzbeziehungen der Synonymie (Heute ist Samstag. - Heute ist Sonnabend.) wie die Oppositionsbeziehungen der Antonymie (Das Wasser ist zu heiß. - Das Wasser ist zu kalt.), die hierarchischen Beziehungen sowohl der Hypo- und Hyperonymie mit Ober- und Unterbegriffen (Er schenkt ihr eine Blume. - Er schenkt ihr eine Rose.) wie auch der Partonymie mit ihrer Teil-GanzesRelation (Sie hat sich die Hand verletzt. - Sie hat sich den Finger verletzt.). Während Kinder noch stark syntagmatisch assoziieren, dominieren bei Erwachsenen die paradigmatischen Assoziationen (CARTER 1987: 158). Die Wahrnehmung eines gemeinsamen Auftretens von Lexemen und dessen Übernahme in das eigene Ausdrucksverhalten sind lernpsychologisch offenbar weniger komplex als die Speicherung einer ganzen Reihe von Lexemen mit gemeinsamen Merkmalen, unter denen ausgewählt werden muss.

Synonymierelationen im Wortschatz sind relativ früh untersucht und in die Theorie vom „Wortfeld“ (zu deren Geschichte und Weiterentwicklung LUTZEIER 1995) aufgenommen worden. Auch wenn diese Theorie in ihrer ursprünglichen strengen Form (lückenloses Abdecken des gesamten Bedeutungsbereichs eines Feldes durch die zugehörigen partiell synonymen Lexeme, die sich semantisch wechselseitig begrenzen) heute nicht mehr vertreten wird, so stellt sie doch eine Hypothese dar, deren Grundgedanke weiterhin gültig ist: Die Lexeme werden im mentalen Lexikon nicht isoliert gespeichert, sondern stets zusammen mit bedeutungsverwandten „Nachbarn“, mit denen sie ein strukturiertes System sich gegenseitig beeinflussender Elemente bildet. Beim sprachlichen Handeln ist die Synonymierelation insofern von zentraler Bedeutung, als beim Abruf eines Lexems durch den Sprecher/Schreiber sich sogleich mehrere bedeutungsähnliche Lexeme einstellen, die eine Auswahl ermöglichen und damit eine besonders präzise, der Situation und dem Kontext angemessene Ausdrucksweise. 
Ulrich, W. - Zur lexikalischen Semantik des Deutschen

Im Übrigen scheint es so $\mathrm{zu}$ sein, dass die Wahrnehmung und die kognitive Verarbeitung von Welt bestimmte Ordnungsmuster vorgibt oder wenigstens nahe legt, die bei der sprachlichen Kommunikation zu einer Begrifflichkeit mit paradigmatischen Beziehungen führt. Z. B. gibt es eine starke, möglicherweise universale menschliche Neigung, ,aufgrund binärer Kontraste zu kategorisieren“ (LYONS 1980: 281) und in „Gegensätzen“/Oppositionen (mit möglicher Verneinung) zu denken und zu sprechen: nicht viel, sondern wenig, heiß kalt, kaufen - verkaufen, männlich - weiblich, ankommen - abfahren, links - rechts usw. Auch gibt es „einen gewissen Grad von hierarchischer Organisation [in großen Teilen des Weltwissens und] in allen Bereichen des Wortschatzes der Sprachen“ (EBD.: 310). Das weist auf eine menschliche Neigung hin, sich einmal präziser, das andere Mal allgemeiner auszudrücken, also merkmalreiche Lexeme wie Rose, Tulpe, Aster, Lilie, Narzisse zu verwenden oder aber diese als koordinierte Unterbegriffe/Kohyponyme unter einem merkmalärmeren Oberbegriff Blume (Hyperonym) zusammenzufassen; es weist ebenfalls darauf hin, Unterscheidungen zwischen einem Ganzen wie Hand und Mantel einerseits, Teilen dieses Ganzen wie Finger und Daumen bzw. Kragen und Ärmel andererseits zu treffen (Partonymie).

\section{Polysemie und "Bedeutungssterne“}

Das Netzwerk der Lexeme bildet die Makrostruktur des Lexikons. Die Lexeme stehen aber nicht direkt miteinander in Verbindung. Sie weisen vor ihrer Verwendung in einem Kontext ein umfangreicheres „Bedeutungspotential“ auf, sind noch mehr oder weniger mehrdeutig (polysem). Ihre jeweilige Kernbedeutung „strahlt“ (,radiality“ bei Lee 2001: 53ff.) in alle Richtungen Nebenbedeutungen aus (UlRICH 2006, 351-356). Dieses „Lesartennetz“ eines Lexems, graphisch zu veranschaulichen als „Wortstern“ (EBD.: 351) oder „Bedeutungsstern“, bildet die Mikrostruktur des mentalen Lexikons. Über ihre Lesartennetze sind die Lexeme somit nur indirekt miteinander verbunden. Als Beispiel zur Veranschaulichung möge ein kleiner Witz dienen:

Ein Vater macht mit seinem kleinen Sohn einen Waldspaziergang. Als sie an einer Stelle mit Blaubeeren vorüberkommen, fragt der Junge: „Was sind denn das für Pflanzen?“ Antwort des Vaters: „Das sind Blaubeeren.“ Darauf der 
Ulrich, W. - Zur lexikalischen Semantik des Deutschen

Sohn, überrascht und ungläubig auf die Pflanzen und die Früchte daran blickend: „Blaubeeren? Aber die sehen doch rot aus!“ Darauf der Vater: „Das liegt nur daran, dass sie noch grün sind.“

Die Verwirrung ist vollkommen. Blau, rot, grün: die Farbbezeichnungen purzeln scheinbar durcheinander. Tatsächlich aber geht es nur um die Nebenbedeutung <unreif> des Adjektivs grün. Wie aber hängt diese Bedeutung mit der Farbangabe als der Kernbedeutung des Wortes zusammen?

Zusammenhänge erhellend sind manche der im Deutschen geläufigen Kollokationen, also relativ feste Wortverbindungen, in denen grün als Attribut zu einem Substantiv tritt. Sie lassen sich leicht im Unterricht zusammenstellen, wenngleich nicht allen Schülern alle Beispiele geläufig und mit ihrer Bedeutung bekannt sein dürften:

grünes Gras, grüne Hochzeit, grüne Seife, grüne Bohnen, grüne Partei, grüne Witwe, grüner Hering, grüner Junge, grüner Tisch, grüne Seite, grüne Hölle, grüne Welle, grüne Lunge, grüne Grenze, grünes Holz

Welcher Schüler weiß, dass grüne Bohnen anders als weiße oder gelbe Bohnen nicht Bezeichnung für die Samen der Bohnenpflanze allein ist, sondern für Schnittbohnen, die Samen also zusammen mit ihren als Gemüse genutzten fleischigen Hülsen? Warum wird der Tag der Eheschließung grüne Hochzeit genannt? Wieso ist ein unreifer junger Mann ein grüner Junge? Was meinen wir eigentlich, wenn wir beim Fischhändler grüne Heringe verlangen? Und wer denkt heute noch bei grüner Seife nicht an ein grünfarbiges Stück, sondern an bräunlich-gelbe Schmierseife, wie sie früher bei der Wäsche im Haushalt verwendet wurde?

Kann man bei dieser vielfältigen Verwendung des Adjektivs überhaupt noch von einem einzigen Wort sprechen? Wenn ja, was hält die unterschiedlichen Bedeutungen dieses Wortes zusammen? 
Ulrich, W. - Zur lexikalischen Semantik des Deutschen

Die Antwort auf diese Fragen lässt sich nach einigem Nachdenken und Nachschlagen in Wörterbüchern durchaus finden:

Die Farbe grün kennzeichnete in noch naturnäheren Zeiten als heute symbolisch für alle Menschen den Jahresbeginn, den Frühling, die wieder zum Leben erwachende Natur mit grünem Gras und grünen Blättern. Von daher steht das Adjektiv für den Anfang von Vorgängen, für ein frühes Stadium in einem Prozess, damit also auch für den Zustand des noch Unfertigen, Unreifen. Das ist neben der Farbbezeichnung die semantische Klammer, welche die verschiedenen Lesarten zusammenhält. Wer dies erkannt hat, kann leicht den grünen Jungen als noch unreifen Menschen und den grünen Hering als den noch unbehandelten, rohen Fisch verstehen, die grüne Seife als ein noch unfertiges Zwischenprodukt im Seifenherstellungsprozess. Im Blick auf den verheißungsvollen Anfang gilt grün auch als die Farbe der Hoffnung.

Die Farbbezeichnung steht also nur als prototypische Kernbedeutung am Anfang einer dynamischen Bedeutungsentfaltung des Wortes. Wie lässt sich dieser semantische Prozess beschreiben?

Von der im Frühling wieder ergrünenden Natur ausgehend hat sich der Gesichtpunkt, das semantische Merkmal <am Anfang eines Prozesses stehend> in den Vordergrund der Vorstellung und in den Vordergrund der Wortbedeutung gedrängt, sodass das Lexem grün auch die folgenden Lesarten angenommen hat: 1. <frisch, saftig>, 2. <unreif>, 3. <erste(r)>, 4. <unfertig> und 5. <roh, weder gesalzen, noch geräuchert, gekocht, gebraten, eingelegt>. Diese Bedeutungsentfaltung ist zugleich eine Bedeutungsverschiebung: ursprünglich nur begleitende randständige Bedeutungsmerkmale konnten dominant werden, z. B. bei grüner Seife das Merkmal <flüssig>. 
Ulrich, W. - Zur lexikalischen Semantik des Deutschen

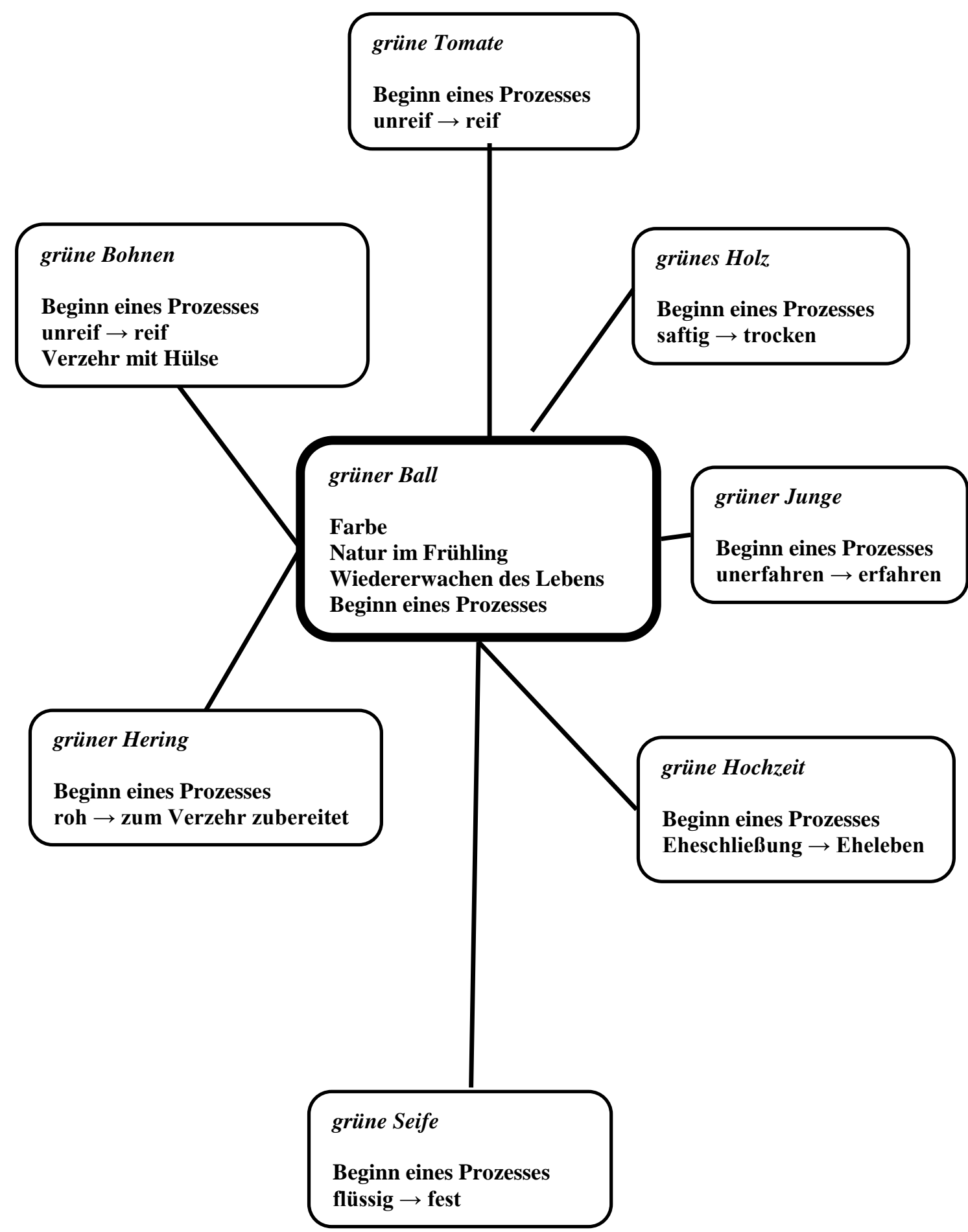


Ulrich, W. - Zur lexikalischen Semantik des Deutschen

Solche Wortsterne lassen sich auch differenzierter entwickeln. Schauen wir uns als zweites Beispiel das vieldeutige Verb gehen in seinen unterschiedlichen Verwendungsweisen an:

1. Er kann schon wieder gehen.

2. Sie werden etwas früher gehen.

3. Er ist deutscher Meister im Gehen über 1000 Meter.

4. Sie geht schon zur Schule.

5. Geh nicht an die Schokolade!

6. Sie geht mit der Mode.

7. Es geht jetzt auf den Winter zu.

8. Wie geht das eigentlich?

9. Mir geht es gut.

10. Das Erbe geht an die Kinder.

11. Das geht wirklich zu weit!

12. Sein Blick geht zur Uhr.

13. Die Uhr geht nicht richtig.

14. Die Geschäfte gehen schlecht.

15. Drei Stück gehen in eine Schachtel.

16. Das Wasser geht ihm bis zu den Knien.

17. Man muss warten, bis der Teig geht.

18. Diese Bemerkung geht auf dich.

19. Hast du gehört, wie die Tür geht?

20. Sein Hobby geht ihm über alles.

21. (Ach) Geh (doch), das ist nicht so schlimm.

22. Sie geht als Nixe zum Fasching.

23. Das geht so leider nicht.

24. Es geht um alles oder nichts.

25. Wohin geht es im Urlaub?

26. Das Fenster geht nach Westen.

Zunächst lässt sich eine prototypische Kernbedeutung beschreiben, als Paraphrase formuliert <sich auf Beinen von einem Ort $\mathrm{zu}$ einem andern fortbewegen>. Merkmalsemantisch müssen mindestens drei Merkmale genannt werden:

$$
\begin{aligned}
& <+ \text { Bewegung }> \\
& <+ \text { von Ort zu Ort }> \\
& <+ \text { auf Beinen }>
\end{aligned}
$$

Bei einer Überprüfung der angeführten Sätze mit dem Verb gehen fällt sofort auf, dass nicht alle Lesarten alle drei Merkmale aufweisen. So können wir schon beim Gehen der Uhr zwar eine Bewegung ausmachen, vielleicht sogar eine von Ort zu Ort (wenn wir auf die Zeiger blicken), aber keinesfalls eine Bewegung auf Beinen. Wenn eine Tür geht, bewegt sie sich, aber nicht an einen anderen Ort, sondern an derselben Stelle, sie wird geöffnet oder 
Ulrich, W. - Zur lexikalischen Semantik des Deutschen

geschlossen. Und wenn jemandem das Wasser bis zum Hals geht, so können wir das Verb durch das partielle Synonym reicht ersetzen; das heißt: die Bewegung des steigenden Wassers ist bereits abgeschlossen, es wird ein erreichter Zustand beschrieben, das Merkmal <Bewegung> ist also allenfalls noch recht blass im Hintergrund der Wortbedeutung vorhanden.

Überhaupt lässt sich beim Vergleich der Lesarten feststellen, dass ihre Beschreibung viel zu grob ausfällt, wenn man nur nach Vorhandensein <+> oder Nichtvorhandensein <-> von Merkmalen fragt. Bei genauerer Betrachtung erkennt man, dass Merkmale innerhalb des semantischen Merkmalbündels eine starke oder eine schwache Rolle spielen können, im Vordergrund oder im Hintergrund stehen. So sind im Satz Geh nicht an die Schokolade die drei Merkmale der Kernbedeutung noch alle vorhanden, allerdings ganz in den Hintergrund gerückt und von einem zusätzlichen Merkmal überdeckt. Zwar muss man sich noch auf die Schokolade zubewegen, wenn sie nicht in Reichweite ist wohl auch auf Beinen, aber viel wichtiger ist, dass man davon einen Teil an sich nimmt <Besitz ergreifen>. Wenn Gäste etwas früher gehen, bewegen sie sich von einem Ort fort zu einem anderen. Das muss nicht auf Beinen geschehen; sie können z. B. mit dem Auto fahren. Entscheidend aber ist, dass sie eine Veranstaltung verlassen. Das Fortgehen wird akzentuiert, nicht das Irgendwohingehen. Und man verlässt nicht irgendeinen Ort, sondern eine Veranstaltung, eine Zusammenkunft mit anderen Menschen.

Das lexikalisch-semantische Netzwerk ist ein dynamisches System, das sich dauernd verändert, wenn Menschen sich bei der geistigen Verarbeitung von Weltwahrnehmungen Vorstellungen/Konzepte bilden und diese dann versprachlichen. Dabei greift man bei ähnlichen Konzepten gern sprachökonomisch auf bereits vorhandene Lexeme zurück und vergrößert die Zahl seiner Lesarten. So kann es zu konzeptionellen Verschiebungen kommen

1. durch Aufgabe vorhandener und/oder Hinzunahme neuer semantischer Merkmale,

2. durch Verrücken von vorhandenen Merkmalen entweder in den Hintergrund (backgrounding) oder in den Vordergrund (foregrounding) (vgl. LEE 2001: 4f.).

So nimmt jemand beispielsweise die Ähnlichkeit zwischen einer Fortbewegung in der Domäne Raum von Ort zu Ort einerseits und der Fortbewegung in der Domäne Zeit von Zeitpunkt zu Zeitpunkt andererseits wahr und formuliert Sie geht mit der Mode; sie geht mit der Zeit. Ausgangspunkt für diese Bedeutungserweiterung durch Domänenwechsel ist die 
Ulrich, W. - Zur lexikalischen Semantik des Deutschen

prototypische Kernbedeutung. Deshalb gibt es auch gemeinsame Merkmale zwischen ihr und der neuen Lesart.

In einem anderen Fall entdeckt jemand eine konzeptionelle Ähnlichkeit zwischen der Fortbewegung eines Menschen in der Zeit und der Bewegung der Zeit selbst, ihrem Verlauf. Dann geht nicht jemand, sondern Es geht auf den Herbst zu. Bei diesem unpersönlichen Gebrauch des Verbs ist das dominante Merkmal <Zeit> die Brücke zwischen einer Lesart und einer anderen. Die Familienähnlichkeit zwischen beiden Lesarten ist größer als die mit der Kernbedeutung.

Noch deutlicher ist dieser Prozess der konzeptionellen Verschiebung und semantischen Distanzierung von der Kernbedeutung bei Verwendung des Verbs in Äußerungen wie Wie geht es dir? und Mir geht es gut. Stark verblasst sind die Merkmale $<$ Fortbewegung> und <Zeit> zwar noch vorhanden, im Vordergrund steht aber ein Hinweis auf die $<$ Verfassung $>$, in der man sich durch das Leben bewegt. Folgt auf die Frage Wie geht es dir? die Antwort Danke, es geht, so haben wir es wohl mit einer Satzellipse zu tun: Danke, es geht mir einigermaßen gut.

<Bewegung> kann auch geistige Prozesse kennzeichnen. So kann man Metaphern als Domänenwechsel interpretieren, z. B. wenn man jemanden auffordert: Geh, stell dich nicht so an! Man fordert dann ja nicht wirklich zum Weggehen auf, sondern zu einer geistigen Bewegung, zur Loslösung vom vorher eingenommenen „Standpunkt“. Und wenn es einmal um viel oder wenig geht, dann handelt es sich um etwas, dann steht etwas auf dem Spiel, d.h. dann ist das Ende einer abstrakten Bewegung, das Ergebnis eines Geschehens offen.

Die ausführliche Einzelanalyse soll hier abgebrochen werden. Verkürzt wird sie aber beim folgenden Versuch einer graphischen Darstellung weitergeführt, mit deren Hilfe die Vernetzung von Kernbedeutung und Lesarten nach dem Radial-Modell veranschaulicht werden soll. Dabei stehen Verbindungslinien für deutliche Familienähnlichkeit und ein bis drei Pluszeichen für abgestufte Relevanz vorhandener semantischer Merkmale: verblasst/backgrounded $\langle+>$, von mittlerer Relevanz $<++>$, dominant/foregrounded $<+++>$. Die Ziffern beziehen sich auf die Nummerierung der Beispielsätze oben. Was hier auf Papier flächig-zweidimensional abgebildet wird, muss man sich dreidimensional mit räumlicher Tiefe vorstellen. 
Ulrich, W. - Zur lexikalischen Semantik des Deutschen

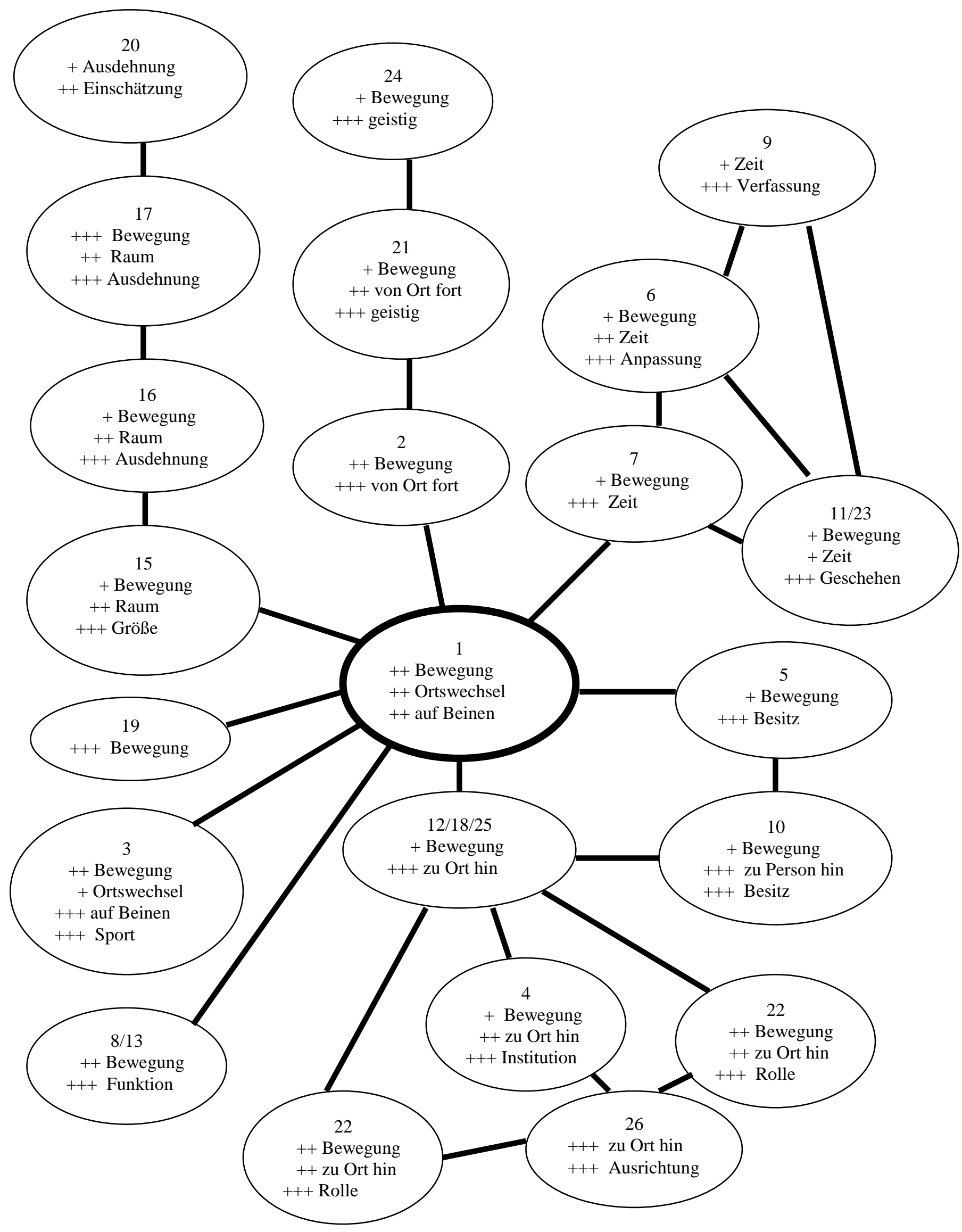




\section{Wortschatzerweiterung und Wortschatzvertiefung}

Untersuchung und Beschreibung der semantischen Strukturen des Lexikons einer Sprache sind insofern von sprachdidaktischer Bedeutung, als eine weniger syntaxzentrierte als vielmehr lexikonorientierte Sprachreflexion, ein „Grammatikunterricht“, der diese Strukturen exemplarisch bewusst macht, sie „nachzeichnet“, in der Lage ist, den Wortschatzerwerb Lernender kräftig zu unterstützen. Das gilt für die quantitative Wortschatzerweiterung, also die Aufnahme neuer, bis dahin unbekannter Lexeme in den Wortspeicher, wie besonders auch für die qualitative Wortschatzvertiefung, bei der weitere Verwendungsweisen, zusätzliche Nebenbedeutungen erlernt werden. ${ }^{2}$

Die folgenden Arbeitsblätter für Schüler vom 5. bis 13. Schuljahr zeigen beispielhaft, wie durch ein „entdeckendes Lernen“ die lexikalischen Strukturen erhellt werden können.

\footnotetext{
${ }^{2}$ Vgl. dazu die einschlägige Monographie des Verfassers, der auch die im Beitrag folgenden Arbeitsblätter entnommen sind: ULRICH, Winfried: Wörter, Wörter. Wörter. Wortschatzarbeit im muttersprachlichen Deutschunterricht. Baltmannsweiler: Schneider Hohengehren, 2. Auflage 2010.
} 
Ulrich, W. - Zur lexikalischen Semantik des Deutschen

\section{Lexikalische Ketten}

\section{trocken}

nass

klitschnass feucht

knochentrocken

1. Versuche die Adjektive so zu ordnen, dass sich eine Steigerung ergibt.

Welches Adjektiv steht ganz am Anfang, welches ganz am Ende der „Wortkette“?

Was nimmt von vorn nach hinten zu?

2. Welches Wort ist das Gegenwort zu groß? Versuche auch für groß und sein Gegenwort Zwischenstufen und Steigerungen zu finden.

Ordne auch diese Wörter so, dass sie sich eine Steigerung oder eine Abnahme der „Größe“" ergibt.

3. Welche Kettenglieder gehören noch zu eiskalt? Ordne sie.

Welche zu blitzschnell?

\section{Lösungen:}

1. knochentrocken $\rightarrow$ trocken $\rightarrow$ feucht $\rightarrow$ nass $\rightarrow$ klitschnass

Der Grad an Feuchtigkeit nimmt zu.

2. klitzeklein $\rightarrow$ winzig $\rightarrow$ klein $\rightarrow$ mittelgro $\beta \rightarrow$ gro $ß \rightarrow$ riesig $\rightarrow$ gigantisch eisig $\rightarrow$ frostig $\rightarrow$ kalt $\rightarrow$ kühl $\rightarrow$ lauwarm $\rightarrow$ warm $\rightarrow$ hei $\rightarrow$ kochendhei $\rightarrow$ gluthei $ß$ langsam $\rightarrow$ gemächlich $\rightarrow$ flink $\rightarrow$ rasch $\rightarrow$ schnell $\rightarrow$ blitzschnell 


\section{Vorangestellte Wortbausteine (Präfixe)}

a) Verbindung, Verständnis, Vertikale, Verlosung, Versprechen, Vergehen

b) Unendlichkeit, Unvollständigkeit, Unverständlichkeit, Untergang, Unschuld

c) Misswahl, Missverständnis, Misshandlung, Missgeschick, Missbrauch

d) Entbindung, Entertainer, Entlastung, Entschuldigung, Entwicklung, Entschädigung

e) Zubereitung, Zubehör, Zusatz, Zugunglück, Zugeständnis, Zuflucht

1. Jede Reihe enthält ein Wort, das nicht hineingehört. Kreise es ein.

2. Wodurch unterscheidet sich dieses Wort von den anderen?

3. Alle Verben in einer der folgenden Reihen können mit dem gleichen Präfix versehen werden. Welches ist es?

a) glühen, glimmen, blühen, hallen, heißen

b) melden, sagen, wiegen, wählen, bestellen

c) klingen, strahlen, glänzen, würgen, glühen

d) schneiden, beißen, knüllen, hacken, reißen

4. Ordne den vier Reihen die folgenden Bedeutungsmerkmale zu:

Vorsicht: In jeder Reihe befindet sich „ein Kuckucksei“. Markiere es!

- Eintreten eines Geschehens

- Objekt in Teile zerlegen

- Ende eines Geschehens

- Geschehen rückgängig machen

5. In der folgenden Reihe sind mehrere Präfixe möglich. Suche mindestens drei.

Auch hier gibt es ein Kuckucksei, wenn du auf die Bedeutungsmerkmale achtest!

machen, kommen, schalten, stellen, brennen

\section{Lösungen:}
a) Vertikale b) Untergang c) Misswahl d) Entertainer e) Zugunglück
Es hat kein Präfix.
a) ver- b) ab-c) er- d) zer-
$\mathrm{cd}$ a b
an-, aus-, ab-, ein-, ver- 


\section{Was wir tun, wenn wir reden}

1. Verbinde jeweils einen Satz aus der ersten Spalte mit einem passenden Satz aus der zweiten Spalte!
a) Reinigen Sie Ihre Uniform?
b) Ich bin unschuldig!
c) Guten Tag, Herr Krause!

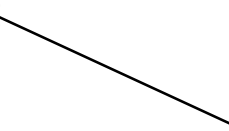
o) Das versichere ich Ihnen.
p) Das rate ich dir.
d) Morgen bekommst du dein Geld zurück.
q) Ich verspreche es dir.
e) Sie bekommen auf jeden Fall eine Entschädigung.
r) Das schwöre ich!
f) Hiermit endet meine Mitarbeit in dem Betrieb.
) Das befehle ich Ihnen!
g) Danke! Danke!
t) Ich gratuliere dir.
h) Vertrag dich mit deiner Schwester.
u) Das prophezeie ich euch.
i) Herzlichen Glückwunsch!
v) Dazu ernenne ich dich.
j) Du wolltest dich bei der Polizei melden.
w) Ich warne dich.
k) Das sollt ihr bereuen!
x) Ich bitte dich darum.
l) Du bist jetzt mein Stellvertreter.
y) Ich danke dir!
m) Der Krieg wird verloren werden.
z) Ich grüße Sie!
n) Vorsicht, gleich kippt es um!
aa) Ich kündige.
bb) Seht es als eine Drohung an.

2. Versuche das Verhältnis zwischen dem einen und dem anderen Satz zu bestimmen. Achte dabei besonders auf die kursiv gedruckten Wörter.

3. Im Folgenden musst du die wörtliche Rede vertauschen, damit die Sätze Sinn ergeben.

a) Er droht ihm: n) „Das hast du gut gemacht!“

b) Er erinnert ihn daran: o) „Verschwinde!““

c) Sie warnt ihn: p) „Du bist ein Esel!“

d) Er fordert sie auf: q) „Du wirst noch dein blaues Wunder erleben.“

e) Er beschuldigt sie: r) „Das ist doch alles nicht so schlimm. Kopf hoch!“

f) Sie entschuldigt sich: s) „Ich werde auf jeden Fall da sein.“

g) Sie beleidigt ihn: t) „Es tut mir leid.“

h) Er ermuntert ihn: u) „Willkommen in Schleswig-Holstein!“

i) Sie lobt sie: v) „Das ist sehr gefährlich.“

j) Er gesteht ihr: w) „Ihr dürft nach Hause gehen.“

k) Er garantiert ihm $x$ ), „Ich habe dich belogen.“

l) Sie erlaubt ihnen: y) „Vergiss nicht, du musst noch zahlen.“

m) Er begrüßt sie: z) „Du hast gestohlen!“

\section{Lösungen:}

1. b-r, c-z, d-q, e-o, f-aa, g-y, h-x, i-t, j-p, k-bb, l-v, m-u, n-w

2. Die kursiv gedruckten Wörter bezeichnen die sprachliche Handlung des ersten Satzes mit einem Wort.

3. a-q, b-y, c-v, d-o, e-z, f-t, g-p, h-r, i-n, j-x, k-s, l-w, m-u 


\section{Metapher - Metonymie}
o Stoß dir nicht den $\mathrm{K}$ o p f an dem Türrahmen!
o Er war der $\mathrm{K}$ o p f der Bande.
o Die Bevölkerung erhält pro K o p f einen Sack Reis.

1. Es handelt sich in den drei Sätzen ja wohl um sehr verschiedene „Köpfe“! Worin bestehen die Unterschiede?

2. Uneigentliche Ausdrucksweise ist dadurch gekennzeichnet, dass man einen vorhandenen sprachlichen Ausdruck nicht verwendet, sondern ihn durch einen anderen ersetzt. Für welche Ausdrücke steht Kopf im zweiten Satz und im dritten Satz?

3. Vergleiche die drei Sätze oben mit den folgenden Satzgruppen.

Lass dich dabei nicht durch die Reihenfolge verwirren.

1. Die Tasse ist auf die Erde gefallen und zerbrochen.

2. Du bist aber eine trübe Tasse!

3. Eine Tasse trinke ich noch.

4. Wir alle leben letztlich doch vom Bauern.

5. Der Bauer pflügt seine Felder.

6. Ich schlage den Bauern mit meinem Turm.

7. Gehöre ich etwa schon zum alten Eisen?

8. Im Kampf auf Leben und Tod stieß er ihm das Eisen in die Brust.

9. Im Laufe der Zeit wird das Eisen rosten.

4. In welchen Sätzen ist ein Wort aus seinem ursprünglichen Sinnbereich in einen ganz anderen Sinnbereich übertragen worden (Metapher)?

Aufgrund welcher Ähnlichkeitsbeziehung ist das geschehen?

Erkläre die Unterscheidung zwischen bildspendendem Bereich und bildempfangenden Bereich.

5. In welchen Sätzen ist ein Wort durch ein anderes ersetzt worden, das in einem sachlichen oder geistigen Zusammenhang mit ihm steht (Metonymie)?

Bestimme diesen Zusammenhang möglichst genau.

Lösungen: 1. Körperteil, Führer einer Gruppe, Einzelperson in größerer Menge. 2. Für Person in bestimmter Rolle bzw. für Person als Mitglied einer Gesamtheit. 3. und 4. Tasse: Bildspendender Bereich ist Tasse mit trübem Inhalt - bildempfangender Bereich ist Gemüt eines Menschen. Bauer: niedriger Stand in Gesellschaftshierarchie - schwache Schachfigur. Eisen: überflüssiger Schrott - alter Mensch ohne anerkannte Leistungen. 5. 3,4,8. Tasse steht für den Inhalt, Bauer für Nahrungsmittel erzeugende Landwirtschaft, Eisen für Waffe aus dem Material. 
Ulrich, W. - Zur lexikalischen Semantik des Deutschen

\section{Nicht immer nur ,machen“"}

1. Formuliere die folgenden Sätze so um, dass das Allerweltswort „machen“ nicht mehr vorkommt.

a) Wer macht heute Essen, du oder ich?

\section{Wer kocht heute, dwoder ich?}

b) An Omas Geburtstag machen wir ein großes Fest!

c) Am 1.April machen die Kinder den Eltern oft einen Streich.

d) In meiner Freizeit mache ich gerne Gedichte.

e) Das macht doch keinen Sinn!

f) Alle Kinder machen jetzt einen Kreis.

g) Mein Sohn hat die Sandburg ganz allein gemacht.

h) In den Ferien mache ich einen Fortbildungskurs.

i) Machst du mir ein Brot?

j) Die Indianer haben mit den Weißen Frieden gemacht.

k) Du hast dir aber deine Haare heute schön gemacht!

l) Wer macht im neuen Stück eigentlich den Romeo?

2. Vergleiche die Sätze miteinander. Welche Formulierungen bevorzugst du?

\section{Lösungen:}

b) feiern, c) einen Streich spielen, d) schreiben, e) ergeben, f) bilden, g) bauen, h) besuchen, i) schmieren, j) schließen, k) frisieren, 1) spielen 


\section{Besondere Beziehungen}

a) Nadel und Faden wie Briefumschlag

$\begin{array}{llll}\text { Stempel } & \text { Papier } & \text { Brief } & \text { Briefmarke }\end{array}$

b) kaufen und verkaufen wie kommen und

sich nähern erscheinen gehen bevorstehen

c) Theater und Schauspielhaus wie Universität und

$\begin{array}{llll}\text { Lehranstalt Hochschule } \quad \text { Akademie } & \text { Wissenschaft }\end{array}$

d) früher und später wie davor und

daneben dahinter nachher vorher

e) Kopf und Haupt wie Pferd

$\begin{array}{llll}\text { Ross } & \text { Schimmel } & \text { Gaul }\end{array}$

f) sitzen und setzen wie liegen und

$\begin{array}{llll}\text { stehen } & \text { legen } & \text { stellen } & \text { ruhen }\end{array}$

g) kalt und eiskalt wie rot und

$\begin{array}{llll}\text { rötlich } & \text { grellrot } & \text { feuerrot }\end{array}$

h) können und Fähigkeit wie müssen und

$\begin{array}{llll}\text { Erlaubnis } & \text { Notwendigkeit } \quad \text { Bwang }\end{array}$

i) flüstern und schreien wie bummeln und

$\begin{array}{lll}\text { rasen } & \text { schleichen } & \text { schlendern }\end{array}$

j) anziehend und anzüglich wie bäuerlich und

ländlich erbaulich bäuerisch bauernschlau

1. Umkreise das richtige Wort.

2. Versuche die Beziehungen zwischen den Bedeutungen der Wörter zu erläutern.

Lösungen: a) Brief, b) gehen, c) Hochschule, d) nachher, e) Ross, f) legen, g) feuerrot, h) Zwang, i) rasen, j) bäuerisch 
Ulrich, W. - Zur lexikalischen Semantik des Deutschen

\section{Literaturverzeichnis}

Aitchison, J. Words in the Mind. An Introduction to the Mental Lexicon. Oxford: Blackwell. - Deutsche Übersetzung (1997): Wörter im Kopf. Eine Einführung in das mentale Lexikon. Tübingen: Niemeyer, $1994^{2}$

CARTER, R. Vocabulary. Applied Linguistic Perspectives. London: Allen \& Unwin, 1987

Clark, E. V. The Lexicon in Acquisition. Cambridge: C.U.P., 1993.

Clark, E. V. Later Lexical Development and Word Formation. In: Fletcher / MacWhinney, B. (eds.) The Handbook of Child Language. Oxford: Blackwell, 1995. S. 393-412.

CRuse, D. A. Lexical Semantics. Cambridge: C.U.P., 1986.

CRuse, D. A. Meaning in Language. An Introduction to Semantics and Pragmatics. Oxford: O.U.P., 2000.

Eisenberg, P. / Linke, A. Wörter. In: Praxis Deutsch 23, 1996, Heft 139. S. 20-30.

EngelKamp, J. Mentales Lexikon: Struktur und Zugriff. In: Harras, G. (Hrsg.). Die Ordnung der Wörter. Kognitive und lexikalische Strukturen. Berlin: de Gruyter, 1995. S. 99-119.

Fletcher, P. / MacWhinney, B. (eds.). The Handbook of Child Language. Oxford: Blackwell, 1995.

GIPPER, H. Kinder unterwegs zur Sprache. Düsseldorf: Schwann, 1985.

HARRAS, G. (Hrsg.) Die Ordnung der Wörter. Kognitive und lexikalische Strukturen. Berlin: de Gruyter, 1995.

HASS, U. Wortschatz und Wortschatzforschung. In: Der Deutschunterricht 1/2006. 2-8.

Hatch, E. / Brown, C. Vocabulary, Semantics and Language Education. Cambridge: C.U.P., 1995.

JESCHENIAK, J. D. Sprachproduktion. Der Zugriff auf das lexikale Gedächtnis beim Sprechen. Göttingen: Hogrefe, 2002.

KAUSCHKE, C. Der Erwerb des frühkindlichen Lexikons: eine empirische Studie zur Entwicklung des Wortschatzes im Deutschen. Tübingen: Gunter Narr, 2000.

KÜHN, I. Lexikologie. Eine Einführung. Tübingen: Niemeyer, 1994.

KÜHN, P. (Hrsg.) Wortschatzarbeit in der Diskussion. Hildesheim: Olms, 2000.

LEE, D. Cognitive Linguistics. An Introduction. Oxford: Oxford University Press, 2001.

LÖBNER, S. Semantik. Eine Einführung. Berlin: de Gruyter, 2003.

LutZeIER, P. R. Lexikologie. Ein Arbeitsbuch. Tübingen: Stauffenburg, 1995.

LYONS, J. Semantik. Band I. München: Beck, 1980. 
Ulrich, W. - Zur lexikalischen Semantik des Deutschen

Murphy, M. L. Semantic Relations and the Lexicon. Antonymy, Synonymy, and Other Paradigms. Cambridge: Cambridge University Press, 2003.

NAtion, I. S. P. Learning Vocabulary in Another Language. Cambridge: Cambridge University Press, 2001.

NeVELING, C. Wörterlernen mit Wörternetzen. Tübingen: Gunter Narr, 2004.

POHL, I. / UlRICH, W. (Hrsg.): Wortschatzarbeit. = DTP 7. Erscheint 2010.

RöMer, C. / MAtzKe, B. Lexikologie des Deutschen. Eine Einführung. Tübingen: Gunter Narr, 2003.

Rothweiler, M. / MeIBAuer, J. Das Lexikon im Spracherwerb. Ein Überblick. Tübingen: Francke, 1999.

SAEED, J. I. Semantics. Oxford: Blackwell, 1997.

Schwarz, M. / Chur, J. Semantik. Ein Arbeitsbuch. Tübingen: Gunter Narr, 1993.

SZAGun, G. Bedeutungsentwicklung beim Kind. Wie Kinder Wörter entdecken. München: Urban \& Schwarzenberg, 1983.

SzAGUN, G. Sprachentwicklung beim Kind. Eine Einführung. München: Beltz, 1996. ${ }^{6}$

TRACY, R. Sprachliche Strukturentwicklung. Linguistische und kognitionspsychologische Aspekte einer Theorie des Erstspracherwerbs. Tübingen: Niemeyer, 1991.

ULRICH, W. Erweiterung des rezeptiven und des produktiven Wortschatzes im muttersprachlichen Unterricht. In: Vocabulary learning in a foreign language Apprendre les mots en langue étrangère - Wörter lernen in der Fremdsprache. (= Triangle 16). British Council, Goethe-Institut, ENS, Paris 1999. 73-89.

UlRICH, W. Wortschatzerweiterung und Wortbildungskompetenz. In: Wortschatz und Wortschatzvermittlung. Hrsg. v. K. DETERING. Lang, Frankfurt a. M.. 2000. (= Folia Didactica 5). S. 9-27.

UlRICH, W. Wortschatzarbeit. In: Didaktik der deutschen Sprache: Ein Arbeits- und Studienbuch in drei Bänden. Texte, Materialien, Reflexionen. 3 Bde. Hrsg. v. W. ULRICH. Klett, Stuttgart 2001. S. 101-191.

UlRICH, W. CD-ROM, DVD, SMS: Vernetzung von Entlehnungen im mentalen Lexikon. Überlegungen zur „Fremdwort“-Didaktik, verdeutlicht am Beispiel der Kurzwörter und Initialwörter. In: Deutschunterricht 57 (2004) S. 34-39.

UlRICH, W.: Lexikologie und Sprachdidaktik. In: Lexikologie. Lexicology. Hrsg. v. D. A. CRUSE / F. HundSNURSCHER / M. JOB / P. R. LUTZEIER. 2. Halbband. (Handbücher zur Sprach- und Kommunikationswissenschaft 21.2). Berlin, New York: de Gruyter. 1875-1879.

UlRICH, Winfried. Wörter, Wörter, Wörter. Wortschatzarbeit im muttersprachlichen Deutschunterricht. Anleitung und praktische Übungen mit 204 Arbeitsblättern in Form von Kopiervorlagen. Schneider Hohengehren, Baltmannsweiler 2007, 2. unveränderte Auflage 2010. ${ }^{2}$ 
Ulrich, W. - Zur lexikalischen Semantik des Deutschen

UlRICH, Winfried (Hrsg.) DTP = Deutschunterricht in Theorie und Praxis. Handbuch zur Didaktik der deutschen Sprache und Literatur in elf Bänden. Baltmannsweiler: Schneider Hohengehren, 2008-2011. 